\title{
Industrial Robotics in Factory Automation: from the Early Stage to the Internet of Things
}

\author{
Antoni Grau ${ }^{1}$, Marina Indri ${ }^{2}$, Lucia Lo Bello ${ }^{3}$, Thilo Sauter ${ }^{4,5}$ \\ ${ }^{1}$ Technical University of Catalonia, Barcelona, Spain, antoni.grau@upc.edu \\ ${ }^{2}$ Politecnico di Torino, Italy, marina.indri@polito.it \\ ${ }^{3}$ University of Catania, Italy, lobello@unict.it \\ ${ }^{4}$ Danube University Krems, Center for Integrated Sensor Systems, Wiener Neustadt, Austria \\ ${ }^{5} \mathrm{TU}$ Wien, Institute of Computer Technology, Vienna, Austria
}

\begin{abstract}
Robotics is a surprisingly old discipline, and robots have shaped industry and the various industrial revolutions for many decades. This paper covers topics relevant to the IES Technical Committee on Factory Automation, focusing in particular on the evolution of industrial robotics. After providing a historical perspective on the topic, the paper addresses current and future trends, revealing the close link between the progress in industrial robotics and the parallel evolution of industrial communication systems, which represent an enabling technology for modern industrial robotics.
\end{abstract}

Keywords-Robotics, industrial communication, Industry 4.0, industrial robotics

\section{INTRODUCTION AND MOTIVATION}

In the last years the increasing spread of smart sensors, able to acquire data from multiple transducers, process it and take the appropriate decisions (such as the activation of one or multiple actuators), has opened new frontiers for the investigation of more and more complex industrial applications. In fact, the capability of such devices to process data in a decentralized way provides a noteworthy potential for distributing the processing activities on multiple nodes to perform complex tasks. Here, communications play a very important role, as they enable the coordination and the cooperation of the actors of automation applications (e.g., sensors, actuators, robotic arms, etc.). Coordinated action of sensors and actuators in the automation field requires network architectures and communication protocols, both wireless and wired, able to support the interaction between multiple devices not only in a reliable way, but also guaranteeing the meeting of the real-time constrains of the supported applications [1]. In this context of distributed processing among coordinated automation devices, teams of cooperating robots come into play. This is a major improvement in robotics, as cooperation enables robots to go beyond the limitations of individuals, providing the possibility to perform more complex tasks [2] than those that each single robot can perform alone.

This paper provides a historical perspective on industrial robotics, from its early stage to the current trends, also looking into the future. The aim is to reveal the close link with industrial communication systems and to discuss the role of advanced communication infrastructures in fostering industrial robotics in the smart factories of the future. The paper is structured as follows: Sect. II provides a survey on the evolution of robotics, while Sect. III describes the parallel evolution of industrial communication systems and its role on the industrial robotics progress. Sect. IV discusses the open issues for the future of robotics. Finally, Sect. V concludes the paper.

\section{The EVOlution OF INDUSTRIAL ROBOtics}

This section presents a historical overview about the origins of industrial robotics and their most typical applications. The fast growth of this field makes it very difficult to forecast the evolution in the long term, and just some current trends are discussed at the end of the section.

\section{A. First Generation}

The term "robot" (Czech for "worker") was coined by K. Capek in 1923, showing the class fighting in a society with automated workers. Isaac Asimov first used the term robotics in science fiction books that inspired scientist and engineers to develop the early industrial robots. At that moment the $3 \mathrm{rd}$ industrial revolution was born. In 1959 George Devol and Joseph Engelberger developed the first industrial robot; it weighed two tons and was controlled by a program on a magnetic drum, with hydraulic actuators and programmed in joint coordinates. In 1961, Unimation installed the first industrial robot used on a production line at the GM Ternstedt plant in Trenton, NJ, which made door and window handles, gearshift knobs, light fixtures and other hardware for automotive interiors. Obeying step-by-step commands stored on a magnetic drum, the Unimate robot's 4,000-pound arm sequenced and stacked hot pieces of diecast metal. The robot cost US\$ 65,000 to make but Unimation sold it for US $\$ 18,000$. In 1962 the first cylindrical robot, 6 Versatran robots were installed by American Machine and Foundry (AMF) at the Ford factory in Canton, USA. It was named the Versatran from the words "versatile transfer".

The first industrial robot in Europe was introduced in 1967, a Unimate, and it was installed at Metallverken, Uppsland Väsby, Sweden. Robot vision, for mobile robot guidance, was demonstrated at the Stanford Research Institute in 1969 with Shakey the Robot [3]. In the same year, Unimate robots 
entered Japanese market signing a licensing agreement with Kawasaki Heavy Industries to manufacture and market Unimate robots in Asia. That year, Hitachi also developed the world's first vision-based fully-automatic intelligent robot that assembles objects from plan drawings. In 1971 the first production line with hydraulic actuated robots at Daimler Benz, Sindelfingen was set. In 1972 robot production lines installed in Europe; FIAT in Italy and Nissan in Japan installed production lines of spot-welding robots. The first fully electric, microprocessor-controlled industrial robot, IRB 6 from ASEA, was presented in 1974. The new inventions, enhancements and improvements have appeared since then year by year.

New architectures took the market with new applications, in 1975 Olivetti set a Cartesian-coordinate robot, first used in assembly applications, in 1978 Hiroshi Makino, from the University of Yamanashi, Japan, developed the SCARA-Robot (Selective Compliance Assembly Robot Arm), and the Programmable Universal Machine for Assembly (PUMA) was developed by Unimation/Vicarm; USA, with support from General Motors [4, 5]. Robotics is applied to new fields that were unthinkable some years ago such the space. Industrials robots are everyday more light, tough, fast and easy to reprogram, with new features to understand their environment, [6], and interact with it and with human in collaborative tasks (co-bots) [7]. Safety is also another important issue in co-bots that is improved day by day $[8,9]$.

\section{B. Traditional Application Domains}

Industrial robots have many applications, and the most representative can be described as follows.

Spot welding involves applying a welding tool to some object, such as a car body, at specified discrete locations. This requires the robot to move its hand (end effector) to a sequence of positions with sufficient accuracy to perform the task properly. It is desirable to move at high speed to reduce cycle time, while avoiding collisions and excessive wear or damage to the robot. Pick and place is the name commonly given to the operation of picking up a part and placing it appropriately for subsequent operations. Pick-and-place operations have some requirements in addition to those for spot welding. The part must not be dropped. It must be held securely enough to prevent it from slipping in the gripper but gently enough to avoid damage. In addition, care must be taken to avoid disturbing the part during approach and departure. Spotwelding and pick-and-place operations are characterized by their point-to-point nature; what happens at the beginning and the end of the motion is critical, but there is some latitude in choosing the intermediate trajectory.

Spray painting requires covering a surface with an even coat of paint. This is typically done by pre-specifying the trajectory along which the arm will move. The trajectory specifies both position and orientation of the nozzle as a function of time. Seam welding requires that a welding torch continuously follow a seam on a surface. Unlike spray painting, seam welding typically requires real-time correction of the path to accommodate small deviations of the actual seam from the expected path. Spray painting and seam welding are both continuous- path applications; position and orientation as a function of time are important throughout the motion.
Electronic testing by robots is being widely used. One application is that of testing the continuity between pins, which involves primarily point-to-point operations. Another application is the detection of flaws in printed circuits by probing along metal traces on circuit boards.

Metrology is now often performed using automated coordinate measuring machines, which are essentially very slow and accurate robots. They are used to measure dimensions of mechanical parts, usually by a sequence of point-to-point motions.

Assembly is an application of increasing importance. Robotic assembly may be done in different ways. One typical method is to equip a simple robot with a special end effector for a particular task, such as inserting a component. The robot is programmed to perform a single operation as a single step in an assembly line. Each robot is fed parts of a single type from a part feeder, which presents them in the correct orientation. In this approach, the robot is used in the same way as hard automation is traditionally used. Feeder mechanisms, which are often quite ingenious, are discussed in [10].

An alternative method is to feed all parts directly into a robot workstation in which the entire assembly is to be completed. Part feeders and magazines may be arranged about the workstation, as may a variety of tools and fixtures required for the assembly. Another option is that the workstation is presented with a "kit" of pre-oriented parts containing all components required for the assembly. To have individual robot workstations do independent assembly of complete products is extremely advantageous for flexible production capacity.

Machining of mechanical parts is a growing application of robotics technology. Operations like grinding, deburring, drilling, milling, polishing, spindling, and sanding parts require the ability to follow surfaces and to maintain the forces required to perform the specified operation.

A number of stringent requirements are imposed upon robots in order for them to be competitive in the world of manufacturing. Reliability and durability are very important. An industrial robot must work every day, often all day, to pay for itself. Accuracy of robots is important for such applications as precise electronic test and for assembly tasks. The ability to comply with the environment is important in assembly and machining applications. Precisely machined parts are usually expensive. Compliant motion is needed to perform adequately with affordable parts. It is highly desirable that robots be sufficiently configurable to allow new sensors to be incorporated. Sensory input should be available to provide continuous servo control and to produce discrete transitions in system behavior [11]. Ease of programming is important, so that robotic applications may be developed quickly. Versatility is needed to avoid the cost of special-purpose fixturing required for new robot workstations. The ability of communicating commands and data provide autonomy to a growing field of mobile robots [12]. Also the use of open source software allows the introduction of ROS into industrial robotics [13]. 


\section{Current Trends}

The prediction of the International Federation of Robotics (IFR, [14]) is that by 2019 more than 1.4 million new industrial robots will be installed in factories around the world. Fig. 1 shows the annual increase of industrial robots supplied from 2000 to 2015. Some trends appear to be boosting robotics industry in the short term. China's economy continues to transition through all the economic stages of industrialization, urbanization and consumption-driven growth. Some factors conduct the growth: cars export requiring a level of quality that can only be provided by utilizing proven robotic automation methods; governmental incentive plans fostering a homegrown robotics industry; rising wages changing the metrics of humanrobot deployment; and general availability of capable factory workers.

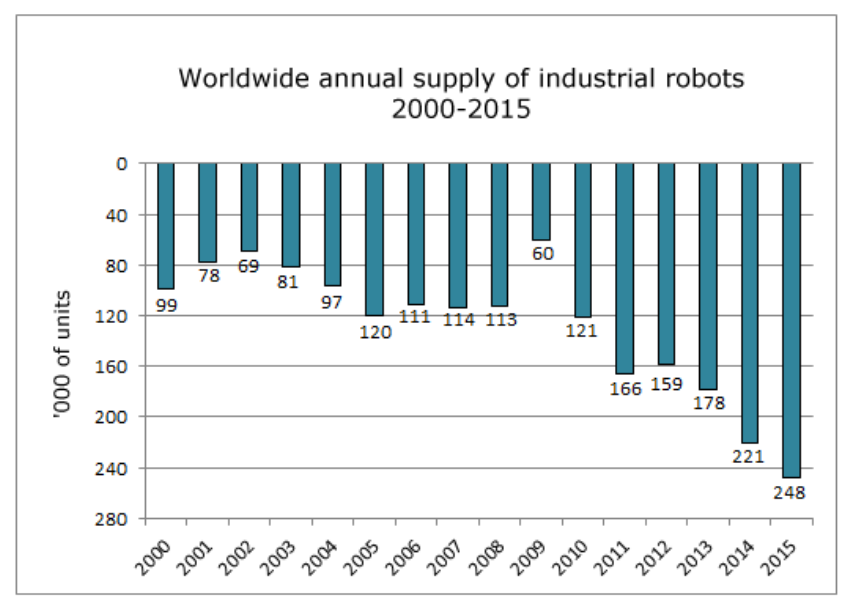

Fig. 1. Evolution of industrial robots supplied from 2000 to 2015. Source: International Federation of Robotics (IFR).

Another trend is collaborative robotics. Car companies replace old-style industrial robots with a combination of humans and co-bots assisting humans to gain needed flexibility. The major benefits of these new co-bots are their flexibility, safety, ability to be rapidly deployed, and ease of training. Turning co-bots into a commodity may not be good for profits but it is good for businesses, particularly those wanting to take their first step into using robots.

Robotics as a Service (RaaS) is another important trend; this concept of offering services instead of the products used in providing the services is and has been a way to introduce untested products into the marketplace but many enterprising startups are finding economies of scale benefit the service provider. The use of unmanned aerial vehicles to capture sensor and camera data and then developing software to analyze that data and translate it into actionable plans has crossed industry boundaries. Better and lower cost vision systems, particularly low-cost 3D vision, navigation and mobility are enabling a variety of existing and startup companies to offer enhanced material handling methods for factories, warehouses and distribution centers. The enormous automation programs with robots had a positive effect on employment not only in the US. In the German automotive sector, the number of employees likewise increased parallel to the growth of robotic automation: The increase between 2010 and 2015 averaged 2.5 percent - the operational stock of industrial robots showed a parallel increase averaging three percent per year, [15]. Reduced production costs result in better market prices. The increasing demand then triggers more jobs.

Experts forecast that as far as technological trends are concerned, companies will, in the future, be concentrating on the collaboration of human and machine, simplified applications, and lightweight robots. Added to this are the twoarmed robots, mobile solutions and the integration of robots into existing environments. There will be an increased focus on modular robots and robotic systems, which can be marketed at extremely attractive prices.

\section{THE ROLE OF INDUSTRIAL COMMUNICATIONS}

The original goal of industrial communication systems was to bridge the manifold gaps in automation systems and to allow for information exchange on the shop floor and beyond. One essential impetus was the recognition in the mid-1970s that since the 1960s, there existed distinctive "automation islands" which employed computers to automatically process data, but that these computerized systems were not interconnected and therefore unable to exchange information. This lack of communication was particularly pronounced on the shop floor, i.e., where the growing complexity of the automation systems called for real-time control of machines are well as their coordination [16]. The first industrial communication systems addressing these specific needs were the so-called fieldbus systems.

By the time fieldbus systems appeared in the late 1980s and 1990 s, robots were already advanced machines, as shown in Fig. 2. Classical fieldbus systems were good enough to provide individual robots with start/stop data or, at most, with some basic set point information. Actual control of the robots was localized, and coordination between robots was not necessary. In those days, robots were mostly part of isolated workstations that were connected via conveyor systems, but at best loosely coupled in a control sense, e.g., by work pieces arriving on pallets which would then trigger the execution of preconfigured manufacturing operations. The limited data transfer and processing capabilities of fieldbus systems could support this kind of structure - and only this kind of structure.

With the introduction of real-time Ethernet (RTE) systems, things did not change too much. This is mostly because RTE was largely seen as a new generation of fieldbus systems based on modern technologies, but not as a new paradigm for industrial communications. RTE does, however, exhibit much larger bandwidth and synchronization accuracy and thus enables applications that were (mostly) not possible with fieldbus systems [17]. In addition, current work within the IEEE Time-Sensitive Networking Working Group is adding new players in the RTE arena. While the IEEE Audio Video Bridging (AVB) standard has already found its way in automotive $\mathrm{A} / \mathrm{V}$ streaming applications [18], the new functionalities provided by the IEEE 802.1Qbu and Qbv standards, such as, preemption and enhanced scheduling for scheduled traffic, are paving the way for deterministic communications over IEEE 802.1Q networks in industrial communications. This opens new scenarios and possibilities 
for a broad spectrum of industrial applications, including industrial robotics, where preemption can be useful to deal with aperiodic activities and so no knowledge about exact traffic arrival patterns is available [19]. RTE variants optimized for motion control can be used inside a robot to coordinate the axes, so that the control of the robot itself can be networked. Moreover, coordination of robots is possible with higher precision because of the possibility to have low jitter synchronization over RTE networks. Finally, the high bandwidth permits inclusion of non-motion-related data such as imaging data or even video streams, which widens the application possibilities.

The situation changed quite significantly with the development of wireless industrial communication systems $[20,21]$. They extended networking into domains that could not be addressed with wired networks. Most obviously, they enabled mobility of network nodes and added ease of installation to the previously rigid communication infrastructure. Recent literature proposed low data rate wireless protocols [22] able to support bounded delays and high scalability in the presence of mobility, while reducing the network overhead and the energy consumption to prolong the network lifetime (for instance, through topology management [23]).

For robotics use cases, this allowed or facilitated the inclusion of mobile devices such as automated guided vehicles (AGVs). Yet, such wireless networks were and are typically only subnets of an otherwise wired infrastructure. Completely wireless networks, on the other hand, are popular among researchers, but still suspicious to operators of manufacturing plants because of dependability issues.

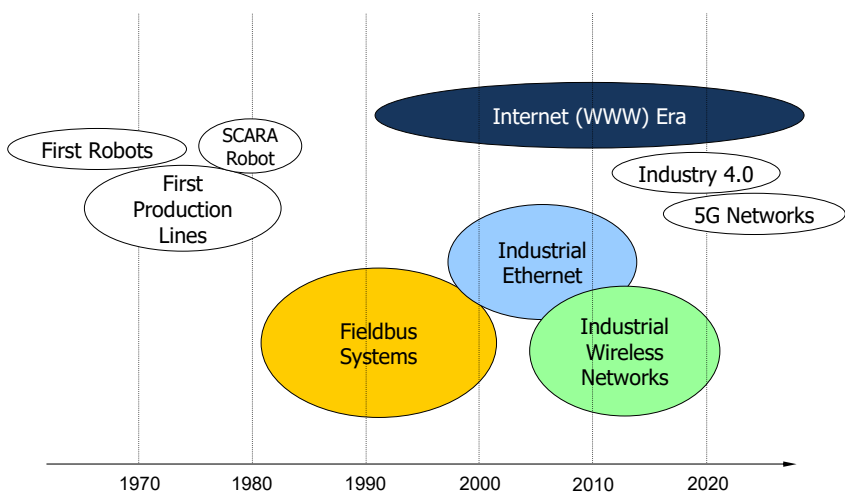

Fig. 2: Essential milestones of industrial communication and robotics.

So far, communication infrastructures in industrial application domains have always been very structured networks. Throughout the evolution, this has not changed much. The recent adoption of IoT and CPS ideas in automation could however initiate a radical change [24]. Both concepts have their origin in the IT world and are based on the observation that today, connectivity is no longer something to desire, but a given fact. The idea is that there is a ubiquitous Internet infrastructure available we can connect to - be it based on mobile networks, WiFi, or wired. This introduces a change of the mindset: Communication is thus taken for granted, and the focus shifts from mere network connectivity to the application, or from mere data transfer to information processing. Consequently, concepts from the IT world as well as consumer electronics are now being applied to automation. The next generation of mobile networks (5G) promises to provide ubiquitous connectivity, and business as well as control logic are moved to some data cloud that intends to leave the frontend devices rather unintelligent. It is still open if this concept will be sustainable and applicable to automation systems with RT demands (such as robotics), but in any case it opens the door for much more flexible, cooperative applications. This is supported by the concept of "digital twins" put forward in the Industry 4.0 framework, where each physical device has a digital counterpart possessing all properties and data of the real-world device. This twin offers services and can connect and interact with other service providers or users. One can envisage that this could be a boost for more advanced robotics applications.

\section{OPEN ISSUES FOR THE FUTURE OF INDUSTRIAL ROBOTICS}

The fourth industrial revolution, which is going to take place in the next years, is expected to deeply change the future manufacturing and production processes, and lead to Smart Factories that will benefit from the main design principles of Industry 4.0 [25]: interoperability, decentralization, real-time capability, virtualization, service orientation, modularity. Robotics will play a key role: innovative technologies and solutions, traditionally associated with the service robotics sector, are going to migrate to industrial smarter robots that will draw on a much broader range of technology, allowing higher levels of dexterity and flexibility, the ability to learn tasks without formal programming, and to autonomously collaborate with other autonomous devices and human operators, thanks to the enhanced capabilities offered by advanced communication networks.

In the academic world, smart robotic applications are being developed and tested every day, but often only through experimental setups built within laboratories, not fully coping with real industrial requirements. On the other side, in the industry world the demand for new, advanced robotic solutions is constantly growing, aiming at smart robotic cells hosting collaborative robots, able to directly cooperate with the human operators, connected through an efficient communication network to intelligent mobile agents, and integrated in an optimized management of the whole production process. At the same time, new application fields are envisaged for mobile and aerial autonomous agents, thanks to the new technological potentialities, e.g., in agricultural and food production scenarios.

The factory of the future scenario relies on the presence and cooperation of both manipulators and mobile agents, sharing spaces with the human operators, directly cooperating with the robots, integrated in an overall communication architecture, allowing an overall optimized handling of all the production processes. Such a factory can be treated as a Cyber-Physical System (CPS) [26], in which robotic systems play an important role, being involved in large productions facilities with different tasks. As discussed in [27], the fractal paradigm can be applied to the factory seen as a complex system of systems, but with relevant self-similarities across the several layers of 
components and structures from the shop-floor up to the enterprise level. The decomposition and distribution of computational processes among the available resources is part of the robotics cloud concept [28], which considers the operation of robots and automation systems relying on data or code from a network, since not all sensing, computation and memory facilities are locally integrated in each single system. The main potential benefits for the robotic agents coming from the cloud are relative to the possibility of accessing wide libraries of images, maps, and trajectories, and to perform efficient parallel computing for learning and motion planning purposes, sharing collective information [29]. The employment of a team of networked robots to flexibly implement a production cycle was already envisaged in [30]. In this scenario, the coordination between robots becomes a key issue to exploit as much as possible the potentialities of a team cooperatively carrying out a common task. Multi-robot coordination addresses several issues, e.g. centralized and decentralized control, formation control, consensus networks, coordinated trajectory tracking, communication infrastructures and resources, but it also multiplies the potential application areas for robotics solutions, starting e.g., from logistics [31] up to data center monitoring [32] and agricultural processes [33]. Interesting solutions in various fields are being developed using mobile robots to create a robotic sensor network, for example as in [34] for pipeline inspection purposes.

One of the most important issue in the new industrial scenario is given by safety, which will have to be guaranteed through proper monitoring and authentication systems [35]. Some monitoring solutions have been proposed in [36] and [37], where the motion of a manipulator is adapted to the presence of the human operator in its workspace on the basis of the information coming from various sensors, like Safety Eye, and possibly Kinect and/or Laser Range Finders. A promising approach for reactive task adaptation has been proposed in [38], where a distributed distance sensor is adopted and integrated within the robot controller to allow safe and task consistent human-robot interaction. All these approaches manage the interaction with a single robot, but in the envisaged scenario more collaborative robots will have to communicate and interact with the environment, with other robots and with human in a dynamic way by taking into account safety and real time constraints. A possible solution for meeting these new challenges relies on the development of a common middleware architecture, e.g., as proposed in [39]. Another question is whether human operators will manage to stay in the loop, keep control and efficiently interact with such "dehumanized" environments. Interaction technologies, design methodologies, intelligent and reliable data fusion and video data analysis are gaining importance for advanced human-machine interfaces in collaborative settings, where robots whose actions are controlled via defined gestures assist human workers [40].

Last but not least, the energy consumption issue is of great importance and it will be even more in the next years. In the past, in the industrial robotics field, energy consumption issues were only sometimes addressed, mainly with reference to specific cases, while in the most recent years a growing interest is shown by several research groups, working on a deeper analysis of the energy consumption of a robot and proposing various approaches to reduce it (see the references in [41]). Energy consumption reduction for a single robot may be achieved in different ways, starting from the trajectory planning phase as in [41], but sustainable productions in the long term could be achieved only via an overall energy optimization of the entire robotic cell (as in [42]), on the basis of efficient optimization procedures running on multicore processors.

\section{CONCLUSIONS AND OUTLOOK}

The paper discussed the evolution of industrial robotics and pointed out the close interplay with wireless industrial communications. Industrial Internet of Things and other technologies that exploit distributed data sensing, gathering and analysis for autonomous decision making enable a level of automation never seen before in industrial robotics. However, the gap between the academic most recent developments in robotics and the organization and working process of most of the current factories is still significant, and should be canceled or at least reduced to speed up the expected renewal of the manufacturing processes. A synergetic academia-industry collaboration may significantly contribute to these purposes, as fostered by the main current research programs (like Horizon 2020), that address as specific challenge the promotion of multi-disciplinary R\&D and innovation activities, like technology transfer via use-cases and industry-academia cross fertilization mechanisms. The aim is to gear up and accelerate cross-fertilization between academic and industrial robotics research to strengthen synergies between their respective research agendas through joint industrially-relevant scenarios, shared research infrastructures and joint small-to medium-scale experiments with industrial platforms. Recent results of these activities make it possible assess the role of advanced communication infrastructures for the most promising topics in the robotics area in the smart factories of the future, like autonomous robotics and mobile robots applications in industrial environments, collaborative robots exploiting advanced sensor fusion capabilities and learning skills, robotic systems in shared spaces, and new smart robotic applications in industrial complex situations and innovative application fields.

\section{REFERENCES}

[1] S. Vitturi, P. Pedreiras, J. Proenza and T. Sauter, "Guest Editorial Special Section on Communication in Automation," IEEE Transactions on Industrial Informatics, vol. 12, no. 5, pp. 1817-1821, Oct. 2016.

[2] G. Patti, G. Muscato, N. Abbate, L. Lo Bello, "Towards Low-datarate Communications for Cooperative Mobile Robots", Proc.. of the IEEE World Conference on Factory Communication Systems (WFCS), Palma de Mallorca, Spain, 05/2015.

[3] SRI website, timeline for computer innovations, https://www.sri.com/work/timeline-innovation, accessed June 5th 2017.

[4] Thomas R. Kurfess, Robotics and Automation Handbook, Taylor \& Francis, 2005.

[5] G. Bekey, J. Yuh, "The Status of Robotics, Report on the WTEC International Study: Part II", IEEE Robotics \& Automation Magazine, DOI: 10.1109/M-RA.2007.907356, March 2008.

[6] S. Y. Chen, "Kalman Filter for Robot Vision: A Survey", IEEE Transactions on Industrial Electronics, vol. 59, no. 11, pp. 4409-4420, Nov. 2012.

[7] A. Gautam, S. Mohan, "A review of research in multi-robot systems", 7th IEEE Int. Conf. on Industrial and Information Systems (ICIIS), pp. 1-5, 6th August 2012. 
[8] M. Vasic, A. Billard, "Safety Issues in Human-Robot Interactions", 2013 IEEE Int Conference on Robotics and Automation (ICRA) Karlsruhe, Germany, pp. 197-204, May 6-10, 2013.

[9] J. Fryman, B. Matthias, "Safety of Industrial Robots: From Conventional to Collaborative Applications", 7th German Conference on Robotics (ROBOTIK 2012), Munich, Germany, May 21-22, 2012.

[10] Sandler, B. Z., Robotics: designing the mechanisms for automated machinery, ${ }^{\text {nd }}$ Edition, Academic Press, 1999.

[11] A. Saudabayev, H. A. Varol, "Sensors for Robotic Hands: A Survey of State of the Art", IEEE Access, Vol. 3, pp. 1765-1782, DOI: 10.1109/ACCESS.2015.2482543, 2015.

[12] Y. Toda, N. Kubota, "Self-Localization Based on Multiresolution Map for Remote Control of Multiple Mobile Robots", IEEE Transactions on Industrial Informatics, Vol. 9, No. 3, pp. 1772-1781, DOI: 10.1109/TII.2013.2261306, 2013.

[13] E. Nieves, "Why industrial robot OEMs should care about ROS", ROScon, Stuttgart, Germany, http://roscon.ros.org/2013/wpcontent/uploads/2013/05/ErikNieves.pdf, 12 May, 2013.

[14] IFR, International Federation of Robotics, press releases website, https://ifr.org/ifr-press-releases/, accessed June 5th 2017.

[15] T. Gregory, A. Salomons, U. Zierahn, "Racing With or Against the Machine? Evidence from Europe", Centre for European Economic Research (ZEW), Discussion Paper No. 16-053, 2017, http://ftp.zew.de/pub/zew-docs/dp/dp16053.pdf

[16] T. Sauter, "The Three Generations of Field-Level Networks-Evolution and Compatibility Issues," IEEE Trans. Ind. Electron., vol. 57, no. 11, pp. 3585-3595, Nov. 2010

[17] M. Felser, "Real-Time Ethernet - Industry Prospective," in Proceedings of the IEEE, vol. 93, no. 6, pp. 1118-1129, June 2005.

[18] L. Lo Bello, "Novel trends in Automotive Networks: A perspective on Ethernet and the IEEE Audio Video Bridging", IEEE Conf. on Emerging Technologies \& Factory Automation (ETFA), Barcelona, Spain, Sep. 2014

[19] G. A. Kaczynski, L. Lo Bello and T. Nolte, "Deriving exact stochastic response times of periodic tasks in hybrid priority-driven soft real-time systems," IEEE Conference on Emerging Technologies and Factory Automation (ETFA), Patras, 2007, pp. 101-110.

[20] S. Vitturi, F. Tramarin, and L. Seno, "Industrial Wireless Networks: The Significance of Timeliness in Communication Systems," IEEE Industrial Electronics Magazine, vol. 7, no. 2, pp. 40-51, June 2013

[21] K. F. Tsang, M. Gidlund and J. Åkerberg, "Guest Editorial Industrial Wireless Networks: Applications, Challenges, and Future Directions," IEEE Transactions on Industrial Informatics, vol. 12, no. 2, pp. 755 757, Apr. 2016.

[22] E. Toscano, L. Lo Bello, "Comparative assessments of IEEE 802.15. 4/ZigBee and 6LoWPAN for low-power industrial WSNs in realistic scenarios", IEEE Workshop on Factory Communication Systems (WFCS), Lemgo/Detmold, Germany, pp. 115-124, May 2012.

[23] E. Toscano, L. Lo Bello, "A topology management protocol with bounded delay for wireless sensor networks", IEEE Conference on Emerging Technologies and Factory Automation (ETFA), 2008, pp. 942-951.

[24] M. Wollschlager, T. Sauter, and J. Jasperneite, "The Future of Industrial Communication - Automation Networks in the Era of the Internet of Things and Industry 4.0," IEEE Industrial Electronics Magazine, vol. 11 , no. 1, 2017, pp. 17-27.

[25] R. Drath and A. Horch, "Industrie 4.0: Hit or Hype? [Industry Forum]," in IEEE Industrial Electronics Magazine, vol. 8, no. 2, pp. 56-58, June 2014.

[26] P. Leitão, S. Karnouskos, L. Ribeiro, J. Lee, T. Strasser and A. W. Colombo, "Smart Agents in Industrial Cyber-Physical Systems," in Proceedings of the IEEE, vol. 104, no. 5, pp. 1086-1101, May 2016

[27] ] M. Pirani, A. Bonci, S. Longhi, "A Scalable Production Efficiency Tool for the Robotic Cloud in the Fractal Factory", IECON 2016, Florence (Italy), 2016

[28] B. Kehoe, S. Patil, P. Abbeel, K. Goldberg, "A Survey of Research on Cloud Robotics and Automation", IEEE Trans. on Automation Science and Engineering, vol. 12, no. 2, pp. 398-409, April 2015
[29] S. Yin, X. Li, H. Gao and O. Kaynak, "Data-Based Techniques Focused on Modern Industry: An Overview," in IEEE Transactions on Industrial Electronics, vol. 62, no. 1, pp. 657-667, Jan. 2015

[30] F. Bullo, J. Cortes, and S. Martinez. "Distributed Control of Robotic Networks: A Mathematical Approach to Motion Coordination Algorithms". Princeton Series in Applied Mathematics. Princeton University Press, 2009

[31] B.Bona, L. Carlone, M. Indri, S. Rosa, "Supervision and monitoring of logistic spaces by a cooperative robot team: methodologies, problems, and solutions", Intelligent Service Robotics, vol. 7, n. 4, pagg. 185-202, 2014

[32] L. O. Russo, S. Rosa, M. Maggiora, B. Bona, “A Novel Cloud-Based Service Robotics Application to Data Center Environmental Monitoring”, Sensors, vol. 16, article 1255, 2016

[33] T. Blender, T. Buchnery, B. Fernandezy, B. Pichlmaiery, C. Schlegel, "Managing a Mobile Agricultural Robot Swarm for a Seeding Task", IECON 2016, Florence (Italy), 2016

[34] D. Wu, D. Chatzigeorgiou, K. Youcef-Toumi and R. Ben-Mansour, "Node Localization in Robotic Sensor Networks for Pipeline Inspection," in IEEE Transactions on Industrial Informatics, vol. 12, no. 2, pp. 809-819, April 2016

[35] F Battaglia, G Iannizzotto, L Lo Bello, "A biometric authentication system based on face recognition and rfid tags", Mondo Digitale 13 (49), 340-346

[36] M. Indri, I. Lazzero, "The RoboLAB experience: aims, challenges and results of a joint academia-industry lab of industrial robotics", $1^{\text {st }}$ Int. Workshop on Robotics Technology Transfer: Innovation from Academia to Industry, 20 $0^{\text {th }}$ IEEE International Conference on Emerging Technologies and Factory Automation (ETFA), Luxembourg, 2015

[37] A. Tellaeche, I. Maurtua, A. Ibarguren, "Human Robot interaction in industrial robotics", $1^{\text {st }}$ Int. Workshop on Robotics Technology Transfer: Innovation from Academia to Industry, 20 $0^{\text {th }}$ IEEE International Conference on Emerging Technologies and Factory Automation (ETFA), Luxembourg, 2015

[38] N. M. Ceriani, A. M. Zanchettin, P. Rocco, A. Stolt and A. Robertsson, "Reactive Task Adaptation Based on Hierarchical Constraints Classification for Safe Industrial Robots," in IEEE/ASME Transactions on Mechatronics, vol. 20, no. 6, pp. 2935-2949, Dec. 2015

[39] H. Fischer, P. Vulliez, J. P. Gazeau and S. Zeghloul, "An industrial standard based control architecture for multi-robot real time coordination," 2016 IEEE 14th International Conference on Industrial Informatics (INDIN), Poitiers, 2016, pp. 207-212

[40] G Iannizzotto, L Lo Bello, "A multilevel modeling approach for online learning and classification of complex trajectories for video surveillance", Int. Journal of Pattern Recognition and Artificial Intelligence, vol. 28, Num. 06, World Scientific Publishing Company

[41] A. Fenucci, M. Indri and F. Romanelli, "An off-line robot motion planning approach for the reduction of the energy consumption", $2^{\text {nd }}$ Int. Workshop on Robotics Technology Transfer: Innovation from Academia to Industry, 21 $1^{\text {st }}$ IEEE International Conference on Emerging Technologies and Factory Automation (ETFA 2016), Berlin, 2016

[42] L. Bukata, P. Šůcha, Z. Hanzálek and P. Burget, "Energy Optimization of Robotic Cells," in IEEE Transactions on Industrial Informatics, vol. 13, no. 1, pp. 92-102, Feb. 2017 